\title{
Iron Fortification of Yogurt and Pasteurized Milk
}

\author{
Smith Gilliard Nkhata*, Zeynep Ustunol and Ahmed Menevseoglu \\ Department of Food Science and Human Nutrition, Michigan State University, USA
}

Received: October 02, 2014; Accepted: April 13, 2015; Published: April 23, 2015

*Corresponding author: Smith Gilliard Nkhata, Department of Food Science and Human Nutrition, Michigan State University, USA, Tel: +265999746608; E-mail: nkhatasmith@yahoo.co.uk

\begin{abstract}
Both yogurt and pasteurized liquid milk was made from whole cow's milk which was fortified with ferrous bisglycinate, ferrous lactate and ferrous sulfate microencapsulate. Yogurt was stored for 7 days and milk for 2 days before consumer acceptance sensory tests was done. Chemical analysis was done every 5 days for yogurt and every 3 days for pasteurized milk. Sensory mean scores show that there were no significant differences between the control yogurt and yogurt fortified with ferrous sulfate microencapsulate in appearance, flavor, mouthfeel and overall preference. Significant differences $(p<0.05)$ were observed between control yogurt and yogurt fortified with ferrous bisglycinate and yogurt fortified with ferrous lactate. The observation was different in milk where no significant differences were observed in appearance and flavor in all treatments while control milk and milk fortified with ferrous lactate showed no significant differences in taste. Both Thiobarbituric Acid (TBA) and Peroxide Value (PV) numbers were highest in yogurt and pasteurized milk fortified with ferrous sulfate microencapsulate. Control yogurt had the lowest TBA value. PV was lowest in yogurt fortified with ferrous lactate while pasteurized milk has low PV in control.
\end{abstract}

Keywords: Fortification; Thiobarbituric acid; Starter culture; Peroxide value; Pasteurization

\section{Introduction}

Iron is essential micronutrient in human nutrition. It is a component of heme in hemoglobin and myoglobin in which it plays important role in the transport, storage and utilization of oxygen. Iron deficiency induces anemia, alters mental development, decreases immunity [1], impairs cognitive scores in children and leads to poor pregnancy outcome [2]. Anemia has been reported to contribute significantly to both maternal and fetal morbidity and mortality [3]. The iron found in food can be highly bioavailable, as is the case with heme iron which is found in red meat. However, the cost of these products is too high for many people. The iron present in other products of vegetable origin, is non-heme and has the disadvantage of interacting with substances in food that inhibit its absorption such as tannins, phytates, and polyphenols hence it has low bioavailability. Much of this kind of food is consumed by people in the lower socioeconomic classes, who cannot meet their physiological needs for iron [3]. Therefore it is widespread in less industrialized countries than in developed countries. The best way to prevent problems associated with iron deficiency is through iron fortification of food for the whole population or only for certain groups. Compounds used in food fortification provide non-heme iron, so it is important to select fortification compounds and foods vehicles that will not diminish iron bioavailability [3]. Though food fortification may increase unit cost of food being fortified, it is the most cost-effective technique than other interventions that have the potential to achieve the same health or nutritional outcome, such as supplementation [4]. Iron fortification of milk and dairy product is considered as a potential approach to prevent the iron deficiency disorder [1], since dairy foods are an important part of the daily diet in most parts of the world. Fortification with iron is technically more difficult than fortification with other nutrients because iron is a pro-oxidant and therefore promotes lipid oxidation [5]. Therefore, the ideal iron compound for food fortification should be one that supplies high bioavailability of iron and does not affect both nutritional value and sensory properties of the food during processing [5].

This necessitates the careful selection of both the food product to be fortified and the iron fortification compound to be added. Clearly, the iron compound must be first optimized with respect to relative bioavailability. Many food vehicles for iron fortification contain substances that inhibit iron absorption. Cereals contain phytic acids and polyphenols, milk contains calcium and caseins while chocolate drinks contain polyphenols [6]. The presence of phytate, the major phosphorus storage compound in grain, has been associated with reduced mineral absorption due to the structure of phyate which has high density of negatively charged phosphate groups which form very stable complexes with mineral ions causing non-availability for intestinal absorption [7]. Polyphenols also forms insoluble complexes with iron thereby reducing its bioavailability to the body. The inhibitory effect of calcium on iron absorption was recognized many years ago. Different studies have been conducted but they often give conflicting results because several factors influence the interaction between calcium and iron absorption [8]. Calcium inhibits absorption of both heme and nonheme. It enters into the mucosal cells by different pathways and leave in the same form which implies that calcium inhibit the intracellular transport of iron [9]. To ensure a level of absorption that is high enough to improve or maintain iron status, it is necessary to prevent the fortification iron from reacting with 
the absorption inhibitors that are inherently present in those foods. This can be accomplished by adding absorption enhancers. The most common enhancer is vitamin C. Alternatives would be bovine hemoglobin and NaFeEDTA where iron is in a protected form. Vitamin $\mathrm{C}$ can increase absorption of both native iron and fortification iron several folds due to both its reducing power and chelating action [6].

\section{Methodology}

\section{Materials}

Whole cow's milk (Michigan Milk Producers Association, Ovid, MI, USA) for yogurt production was provided by Michigan State University Dairy Plant, East Lansing, MI. Milk for production of pasteurized milk was purchased from Bunda College Student Farm, Lilongwe, Malawi. Ferrous bisglycinate, ferrous lactate and ferrous sulfate microencapsulated with $50 \%$ vegetable fats (Dr. Paul Lohmann Inc, US) with the following iron content; 23\%, $19 \%$ and $18 \%$ respectively were used for this study. Commercial Hansen's DVS yogurt culture Yo-fast containing Lactobacillus delbruckii ssp Bulgaricus and Streptococcus thermophilus was purchased from Chris Hansen Milwaukee, WI. MRS agar (Difco, USA) was obtained from Microbiology Laboratory at Michigan State University, MI USA. Chloroform, glacial acetic acid, Thiobarbituric acid (TBA), starch, sodium hydroxide, toluene, sodium thiosulfate (Sigma-Aldrich, USA) were also used. However some of chloroform and glacial acetic acid used for milk analysis were purchased from Lab Enterprises, Blantyre, MW. Additional ingredients that were used to manufacture yogurt were sucrose from Michigan Sugar Company (Saginaw, MI, USA), stabilizer (Continental Custom Ingredients, Chicago, IL, USA), non-fat dry milk solids (Michigan Milk Producers Association, Ovid, MI, USA) and strawberry puree (Kraus \& Co., Walled Lake, MI, USA)

\section{Starter culture growth and activity}

Reconstituted skim milk (12\% w/v) was fortified with ferrous bisglycinate $(63 \mathrm{mg} / \mathrm{kg})$, ferrous lactate $(79 \mathrm{mg} / \mathrm{kg})$ and sulfate dried microencapsulate (83 mg/kg) (Dr Paul Lohmann Inc, US). The fortification levels were different because ferrous bisglycinate, ferrous lactate and ferrous sulfate microencapsulate have different iron content so there was a need to harmonize iron in the final products. Ferrous bisglycinate has approximately $23 \%$ iron. Ferrous lactate and ferrous sulfate microencapsulate have approximately $19 \%$ and $18 \%$ iron respectively. In order to obtain a product that has approximately $15 \mathrm{mg} / \mathrm{kg}$ of iron, which can contribute $20-30 \%$ towards RDA for different categories of people when $200 \mathrm{ml}$ of fortified product is consumed, there was a need to compute how much of each iron salt should be used for fortification. Using same levels of fortification for all iron salts would mean that more iron would be present in one sample and less iron in the other sample. This would undoubtedly affect both the sensory and chemical analysis results. Iron compound was omitted from control treatment. The reconstituted milk was then sterilized at $121^{\circ} \mathrm{C}$ for 5 minutes. Each flask was cooled to $35^{\circ} \mathrm{C}$ and inoculated with $1 \%$ (wt/wt) commercial Hansen's yogurt culture Yo-fast 10, containing Lactobacillus delbruckii ssp bulgaricus and Streptococus thermophilus, and incubated at $35^{\circ} \mathrm{C}$ for 6 hours. Growth of lactic acid bacteria was determined by sampling at 1.5 hour interval and plating on de Man Rogosa, and Sharpe (MRS) agar (Difco). Plates were incubated at $35^{\circ} \mathrm{C}$, 48 hours under aerobic conditions. After incubation the colonies formed were counted and results expressed as $\mathrm{CFU} / \mathrm{ml}$.

\section{Skim milk and yogurt Titratable Acidity (TA)}

Prior to determining Titratable Acidity (TA) the samples were thoroughly mixed. Nine grams of each sample was placed in Erlenmeyer flask to which 4 drops of phenolphthalein indicator was added. The mixture was titrated with $0.1 \mathrm{~N} \mathrm{NaOH}$ to the first permanent shade of pink.

\section{Yogurt manufacture}

Four batches of yogurt were manufactured at Michigan State University Dairy Plant using the following formulation; 80.5\% whole milk, 4.0\% Nonfat Dry Milk (NDM) (Michigan Milk Producers Association, Ovid, MI, USA), 0.5\% stabilizer (Continental Custom Ingredients, Chicago, IL, USA) and 5.0\% sucrose (Michigan Sugar Company, Saginaw, MI, USA). The whole milk was fortified with ferrous bisglycinate $(63 \mathrm{mg} / \mathrm{kg})$, ferrous sulfate microencapsulate $(83 \mathrm{mg} / \mathrm{kg}$ ) or ferrous lactate $(79 \mathrm{mg} / \mathrm{kg})$. Control yogurt had no iron salts added. The yogurt mix was warmed to $60^{\circ} \mathrm{C}$, homogenized dual stage 2000, $500 \mathrm{psi}$ and pasteurized at $85^{\circ} \mathrm{C}$ for $30 \mathrm{~min}$ then cooled to $43^{\circ} \mathrm{C}$. It was inoculated with $1 \%$ (wt/wt) commercial Hansen's yogurt culture Yo-fast 10, containing Lactobacillus delbruckii ssp bulgaricus and Streptococus thermophilus and incubated at $43^{\circ} \mathrm{C}$ until pH 4.6 was reached. Finally, 10\% strawberry puree (Kraus \& Co., Walled Lake, MI, USA) was added as flavorant.

Yogurt for each treatment was divided and stored into two separate containers. One part was used for sensory analysis while the other was used for chemical analysis of lipid oxidation. Yogurt for sensory analysis was separated and stored at refrigeration temperature for one week before sensory evaluation was done in order to give time for the iron to fully interact with milk component. It was feared that if the sensory analysis was done immediately after manufacturing yogurt the sensory results would not reflect the actual sensory attributes of stored yogurt as commercial yogurt spend some time in storage or on shelves before it is bought and consumed by consumers. Yogurt for chemical analysis was also stored at refrigeration temperature and the analysis was done after every 5 days till 35 days storage period was reached. This period was chosen because it approximates the shelf life of yogurt under refrigeration.

\section{Processing of pasteurized milk}

Whole milk (Bunda College Student Farm, Lilongwe, MW) was fortified with ferrous bisglycinate $(63 \mathrm{mg} / \mathrm{kg})$, ferrous lactate $(79 \mathrm{mg} / \mathrm{kg}$ ) and ferrous sulfate ( $83 \mathrm{mg} / \mathrm{kg})$. No iron salts was added to control treatment. Batch pasteurization was used to pasteurize milk from each treatment separately where milk was heated in a container to $63^{\circ} \mathrm{C}$ and held at this temperature for 30 minutes. Thermometer was used to detect temperature changes. While milk was being pasteurized bottles were being heated at temperature around $100^{\circ} \mathrm{C}$ to reduce recontamination 
of pasteurized milk. When the pasteurization temperature and time was reached, the milk was then cooled and packed in bottles and stored under refrigeration temperature. Ten bottles were prepared for each treatment; therefore 40 bottles were prepared for all the four treatments. At each testing interval a single bottle from each treatment was used and the remaining contents were discarded.

\section{Determination of $\mathrm{pH}$}

The $\mathrm{pH}$ meter was used to measure the $\mathrm{pH}$ of the samples. The tip of the $\mathrm{pH}$ meter sits in a buffer which is standardized to a neutral pH 7. Small samples of yogurt were taken from each treatment for measurements. The HI 2210 bench top pH meter with combined glass electrode was inserted in yogurt for few minutes and was read when it stabilized. This was repeated for each sample and cleaning the electrode with distilled water after using on each sample and finally the tip for the electrode was dipped in a buffer solution.

\section{Peroxide value determination}

Yogurt was diluted with distilled water to $20 \%$ solution. Five grams of $20 \%$ yogurt solution was put in $250 \mathrm{ml}$ Erlenmeyer flask to which $30 \mathrm{ml}$ of acetic/chloroform solution was added and swirled in order to dissolve the sample. $0.5 \mathrm{ml}$ of saturated KI was added by pipette and the solution was allowed to stand while occasionally shaken for 1 minute. Thirty $\mathrm{mL}$ of distilled water and $1 \mathrm{~mL}$ starch indicator (1\% starch) was added. $0.01 \mathrm{~N}$ sodium thiosulphate solutions was titrated immediately until the brown (or yellow) color disappeared representing end point. This procedure was repeated for pasteurized liquid milk where $5 \mathrm{~g}$ sample was used in place of $20 \%$ yogurt. The determination on the blank was done on $30 \mathrm{~mL}$ acetic acid/chloroform solution + $0.5 \mathrm{~mL}$ KI solution $+30 \mathrm{~mL}$ distilled water $+1 \mathrm{~mL}$ starch indicator using $0.001 \mathrm{~N}$ sodium thiosulfate as a titrant. The peroxide values were expressed as milliequivalents of peroxide per $1000 \mathrm{~g}$ sample using.

\section{Thiobarbituric Acid (TBA) value}

One gram yogurt was diluted with $100 \mathrm{ml}$ distilled water then $3.0 \mathrm{~g}$ diluted yogurt was put in Erlenmeyer flask (125-300ml) separately, and $10 \mathrm{ml}$ toluene was added to samples separately. Then $10 \mathrm{ml}$ of TBA reagent which was prepared by dissolving $1 \mathrm{~g}$ of TBA powder in $75 \mathrm{ml}$ of $0.1 \mathrm{~N} \mathrm{NaOH}$ and diluted to $100 \mathrm{ml}$ with distilled water according to Sinnhuber and Yu [10], was pipetted (using a pipette bulb) into the flask. The flasks were swirled and shake frequently for four minutes. The entire content was transferred into $250 \mathrm{ml}$ separatory funnel. The layers were allowed to separate and the lower layer was collected in a screw cap test tube $(18 \times 145 \mathrm{~mm}$ or $25 \times 200 \mathrm{~mm})$. The tube was heated in boiling water for 30 minute. The test tube was cooled under running water and transferred the portion of the sample to the Spectronic 20 cuvette. The absorbance of the sample at 530nm was read using distilled water as the blank. Average absorbance $\times 100$ as the TBA values is reported with the result normalized per $g$ of sample.

\section{Sensory evaluation}

Yogurt: Consumer acceptance sensory test was carried out in which four samples of yogurt fortified with ferrous bisglycinate $(63 \mathrm{mg} / \mathrm{kg})$, ferrous lactate $(79 \mathrm{mg} / \mathrm{kg})$, ferrous sulfate microencapsulated $(83 \mathrm{mg} / \mathrm{kg})$ and a control treatment were presented to 100 untrained panelists upon approval by University Committee on Research Involving Human Subject (UCRIHS). The panelists were recruited by posting flyers around Michigan State University (MSU) campus and by sending emails containing the flyer to different departments at MSU. Ninety eight panelists consisting mainly graduate students, undergraduate students and faculty at MSU. Sensory evaluation was conducted in individual booths in the sensory laboratory in the Department of Food and Human Nutrition at MSU. Upon arrival at the sensory laboratory each subject read an explanation of the study and gave their informed consent. Yogurt samples were put into $2 \mathrm{oz}$ plastic cups labeled with randomly selected three digit numbers and refrigerated till time of evaluation. The samples were presented in a randomized manner across subjects to ensure that the order did not introduce bias into the results. Subjects were asked to taste and evaluate all four samples and indicated their degree of liking on a nine point hedonic scale from 1 = dislike extremely to $9=$ like extremely and $5=$ neither like nor dislike. The panelist evaluated each sample for likeability of the appearance, body texture, flavor and overall acceptance. Panelists were provided with purified water at room temperature for rinsing between samples.

Pasteurized milk: Pasteurized milk was manufactured at Bunda College Food Laboratory in the Department of Home Economics and Human Nutrition. The milk was stored for 2 days at refrigeration temperature before consumer acceptance sensory test was done. The milk was evaluated using consumer panel. The panelists were recruited by flyer pasted on different notice boards around Bunda College campus. One hundred panelists consisting primarily of undergraduate students, graduate students and staff members participated in the sensory evaluation. Upon arrival at the food laboratory each subject read an explanation of the study and gave their informed consent. There was not a separate approval for this evaluation because the UCRIHS approval was for both yogurt and pasteurized milk. Fifty millimeters of each sample were put in a hundred millimeters cup labeled with randomly selected three digit numbers. The order of presentation was randomized. Subjects were asked to taste all the four samples and indicate their degree of liking on a nine point hedonic scale from $1=$ dislike extremely to $9=$ like extremely and $5=$ neither like nor dislike. The panelists evaluated the samples based on appearance, mouth feel, taste, flavor and overall acceptance. The panelists were provided with water for rinsing between samples.

\section{Statistical analysis}

Statistical parameters that were generated were mean, standard deviation and one way analysis of variance (ANOVA) for sensory, TA, PV and TBA data. Statistical software IBM SPSS Statistics 20 was used for statistical analysis of the results. For yogurt sensory analysis SIMS 2000 for windows was used to 
generate means and statistical differences. Least Significant Difference (LSD) at $p<0.05$ was used to determine significant differences between means from all the treatments. All experiments were replicated twice except for starter culture growth and activity experiment which was done once with two analyses.

\section{Results}

The growth and activities of commercial Hansen's yogurt culture Yo-fast 10 containing Lactobacillus delbruckii ssp Bulgalicus and Streptococus thermophilus were different from each other amongst treatments. Though at time $0 \mathrm{hr}$ the bacterial count was almost the same for all treatments, there was steady and different growth rates as time progressed. After 6 hours both ferrous bisglycinate and ferrous sulfate had the highest bacterial population indicating that they supported bacterial growth the best as shown in figure 1 . None of the iron salts were inhibitory to the growth of the yogurt culture.

There was a steady increase in TA in all the treatments as time progressed though TA changes were not significantly different between treatments at each testing interval. This means that there was increase in microbial growth and activities which resulted into increased acidity due to activities of lactic acid bacteria; Lactobacillus delbruckii ssp bulgalicus and Streptococus thermophilus as shown in figure 2. Hutkins and Nannen [11] observed that during growth and fermentation the $\mathrm{pH}$ of the medium decreases due to accumulation of organic acid primarily lactic acid.

When yogurt batches were put in the incubator there were noticeable differences in reaching the desirable $\mathrm{pH} 4.6$ which is the isoelectric point of caseins. This isoelectric point is important because milk caseins coagulate to for curd, a characteristic texture of yogurt. The difference in reaching the isoelectric point was due to difference in microbial activities that were taking place during incubation that resulted into a decrease in $\mathrm{pH}$. The $\mathrm{pH}$ was decreasing due to accumulation of lactic acid as bacterial culture was breaking down lactose in order to obtain energy. The treatment fortified with ferrous sulfate was the first

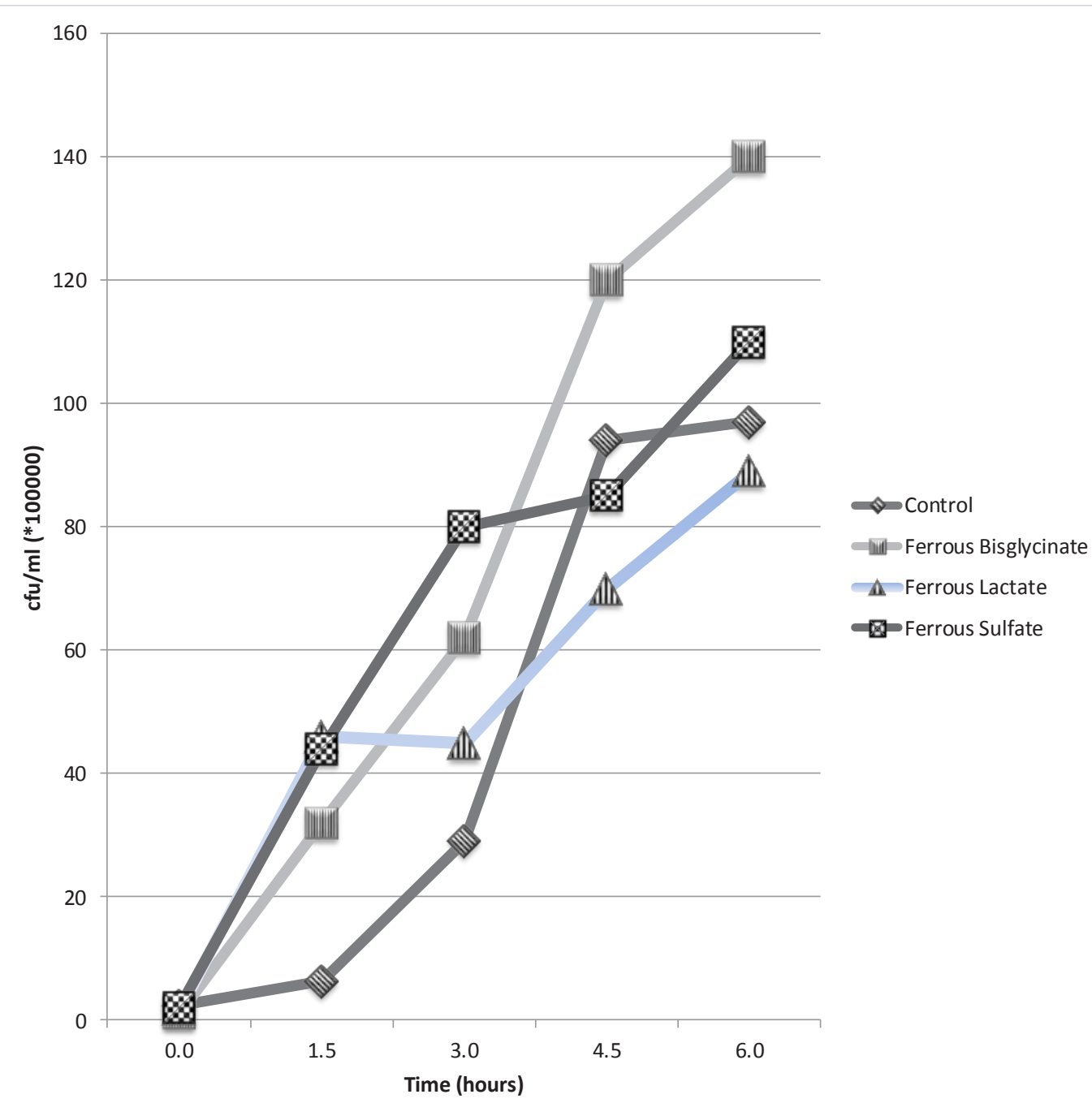

Figure 1: Effects of iron salts on growth and activities of Lactobacillus delbrueckii subsp. bulgaricus and Streptococuss thermophilus in iron fortified reconstituted skim milk incubated at $35^{\circ} \mathrm{C}$ for 6 hours. 


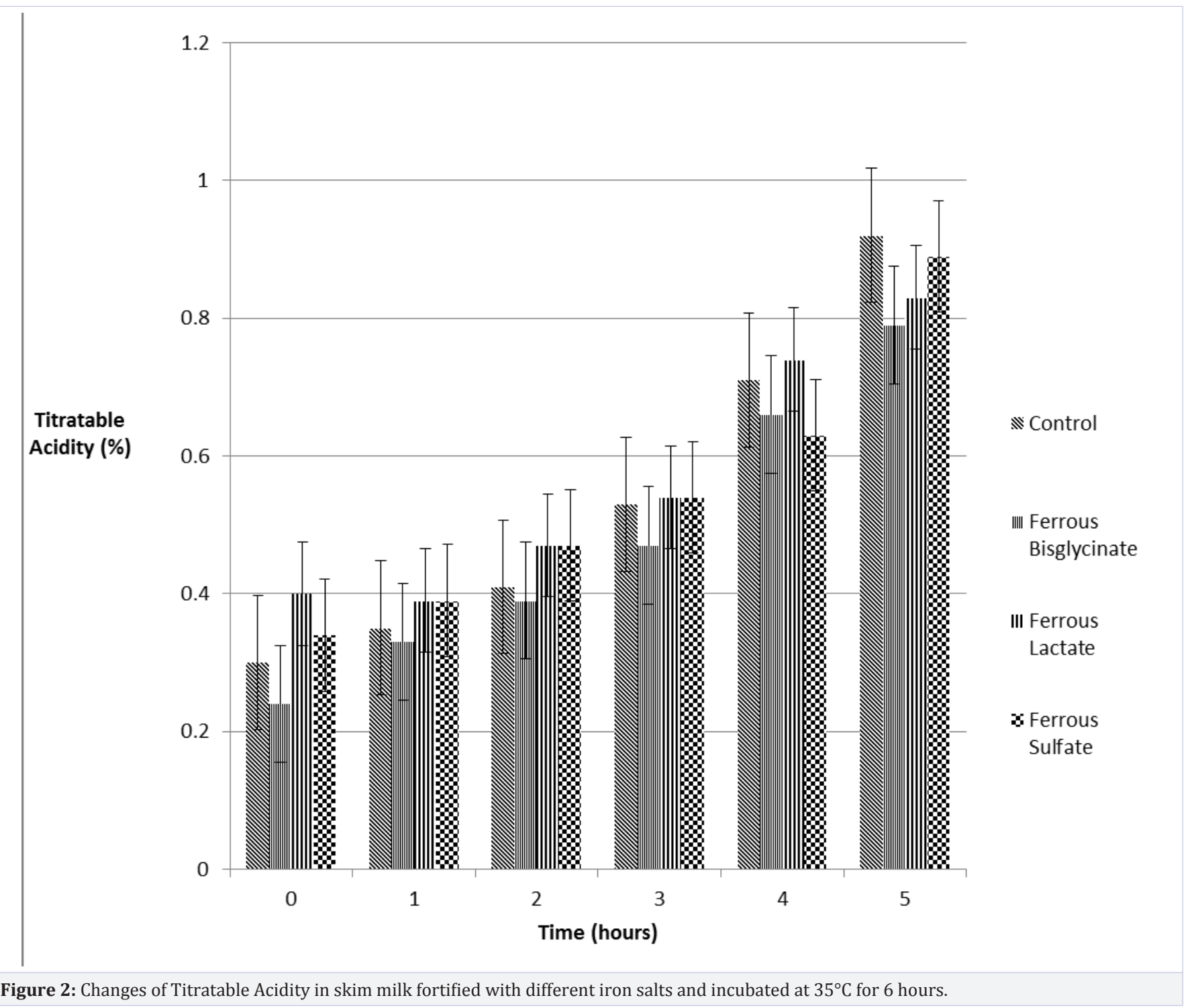

to reach the desirable $\mathrm{pH}$ after 4.5 hours as shown by figure 3 . This observation justifies why ferrous sulfate microencapsulate fortified yogurt showed rapid microbial growth and activities that resulted into decrease of $\mathrm{pH}$. Ferrous lactate was the last to reach $\mathrm{pH} 4.6$ due to low lactic production. Ferrous bisglycinate and ferrous lactate took more than 6.5 hours to reach desirable $\mathrm{pH}$. These differences in reaching the desirable $\mathrm{pH}$ were due to the differences in microbial activities by Hansen's yogurt culture Yo-fast 10, Lactobacillus delbruckii ssp bulgaricus and Streptococus thermophilus.

Results in figure 4 shows that there was a general trend in all the treatments in regard to the TA in stored yogurt. There was an increase in lactic acid expressed as \%TA up to first 15 days. Thereafter there was a decrease in TA. During growth and fermentation, the $\mathrm{pH}$ of the medium decreases because of the accumulation of organic acids, primarily lactic acid. Growth of lactic acid bacteria continues as long as there are enough growth nutrients, no toxic or inhibiting compounds and the hydrogen ion concentration is maintained above the level that the strain can tolerate [11]. The lowest $\mathrm{pH}$ was at day 15, as shown by figure 5, which was due to high lactic acid accumulation (high TA). This high lactic acid became the growth limiting factor for bacteria hence bacterial growth was retarded and eventually the production of lactic acid decreased. This decrease in $\mathrm{pH}$ in fermented food is advantageous in the sense that organic acids produced during yogurt fermentation can potentially enhance iron and zinc absorption via the formation of soluble ligands [12]. Especially in weaning foods, such physicochemical properties of fermented foods is highly desirable, for the fact that children are most of the time highly vulnerable for food pathogens due to their physiological conditions $[13,14]$. The differences in $\mathrm{pH}$ for different iron salts suggest that bacterial activities in stored yogurt were different in each of the treatments. In all cases the $\mathrm{pH}$ for yogurt fortified with ferrous bisglycinate was highest except on day zero. The highest $\mathrm{pH}$ meant that yogurt culture growth was still more active as low $\mathrm{pH}$ was not yet a limiting factor. 


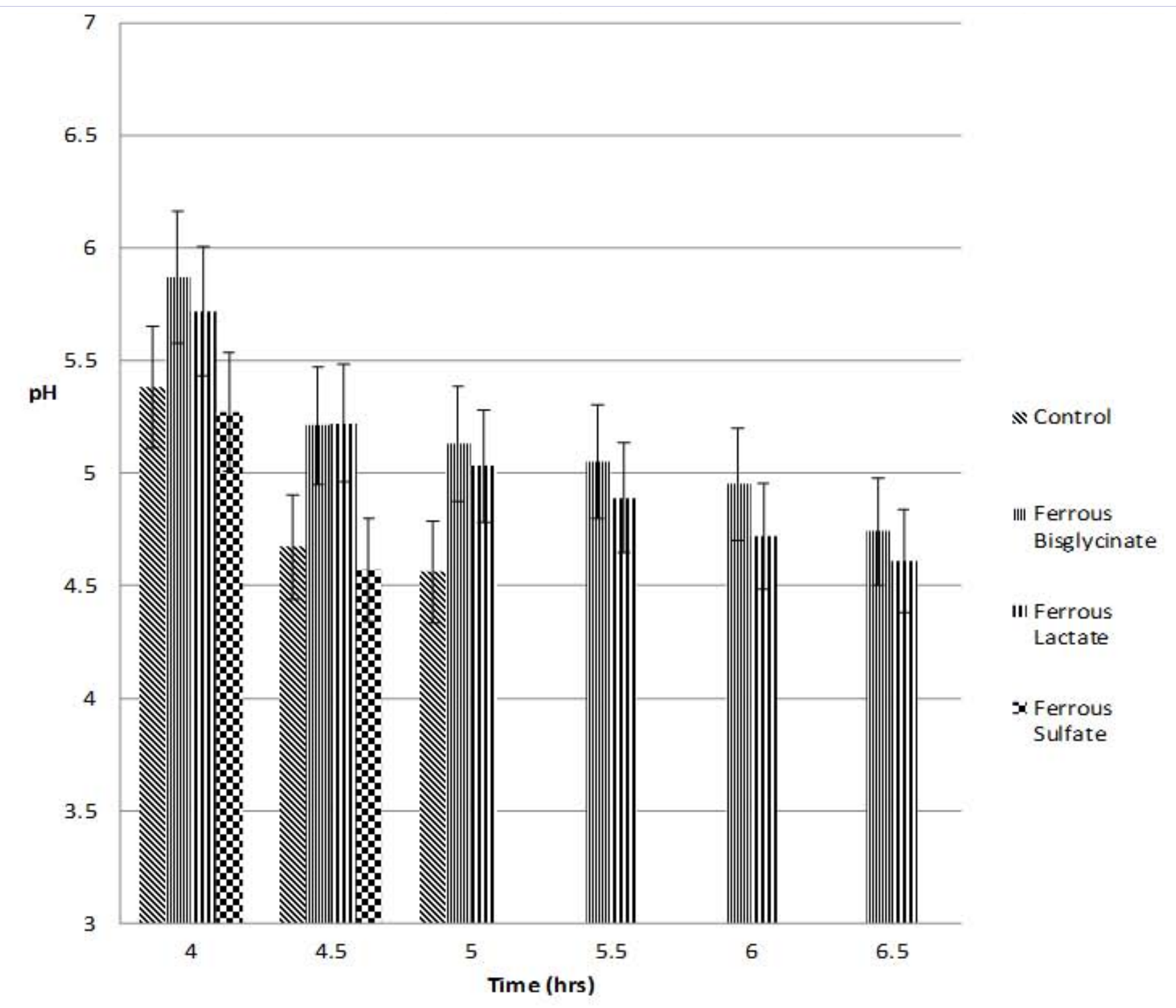

Figure 3: Changes in $\mathrm{pH}$ for yogurt fortified with different iron salts during incubation at $43^{\circ} \mathrm{C}$ till $\mathrm{pH} 4.6$ was reached.

\section{Sensory Analysis}

\section{Sensory evaluation of yogurt}

Data in Table 1 shows that there were significant differences in sensory attributes between samples. There was no significant differences between the control yogurt and yogurt fortified with ferrous sulfate microencapsulated in all the attributes studied $(p<$ $0.05, \mathrm{n}=98$ ). This shows that fortifying yogurt with ferrous sulfate microencapsulate does not affect the consumer acceptability of yogurt. This is a welcome idea considering that the average relative bioavailability of ferrous sulfate when used as a fortificant is $100 \%$ [6]. Both ferrous bisglycinate and ferrous lactate were significantly lower than control treatment in appearance, body texture, flavor and overall acceptance $(p<0.05, \mathrm{n}=98)$. This means that they both affected consumer acceptability of yogurt. Yogurt fortified with ferrous bisglycinate had the lowest flavor and appearance acceptability scores because ferrous bisglycinate easily oxidizes to ferric form $\left[\mathrm{Fe}^{3+}\right]$ which then causes off-color development and fat oxidation [15]. Yogurt fortified with ferrous bisglycinate and ferrous lactate scored significantly lower than control yogurt because panelists said these iron salts made yogurt grayish which was unpleasant to the panelists. While the color of ferrous lactate is white the brown appearance of ferrous lactate fortified yogurt maybe due to other reactions between ferrous lactate and milk components. Use of strawberry puree lessened the degree of browning in all yogurts but it was not enough to mask ferrous bisglycinate and ferrous lactate. Encouragingly, all yogurts were rated above average on Hedonic scale. As a result it can be concluded that lipid oxidation that was detected by PV and TBA resulting from iron fortification had a negligible effect on how well the yogurts were liked. Yogurt fortified with ferrous bisglycinate had significantly higher $\mathrm{pH}$ at each testing interval 


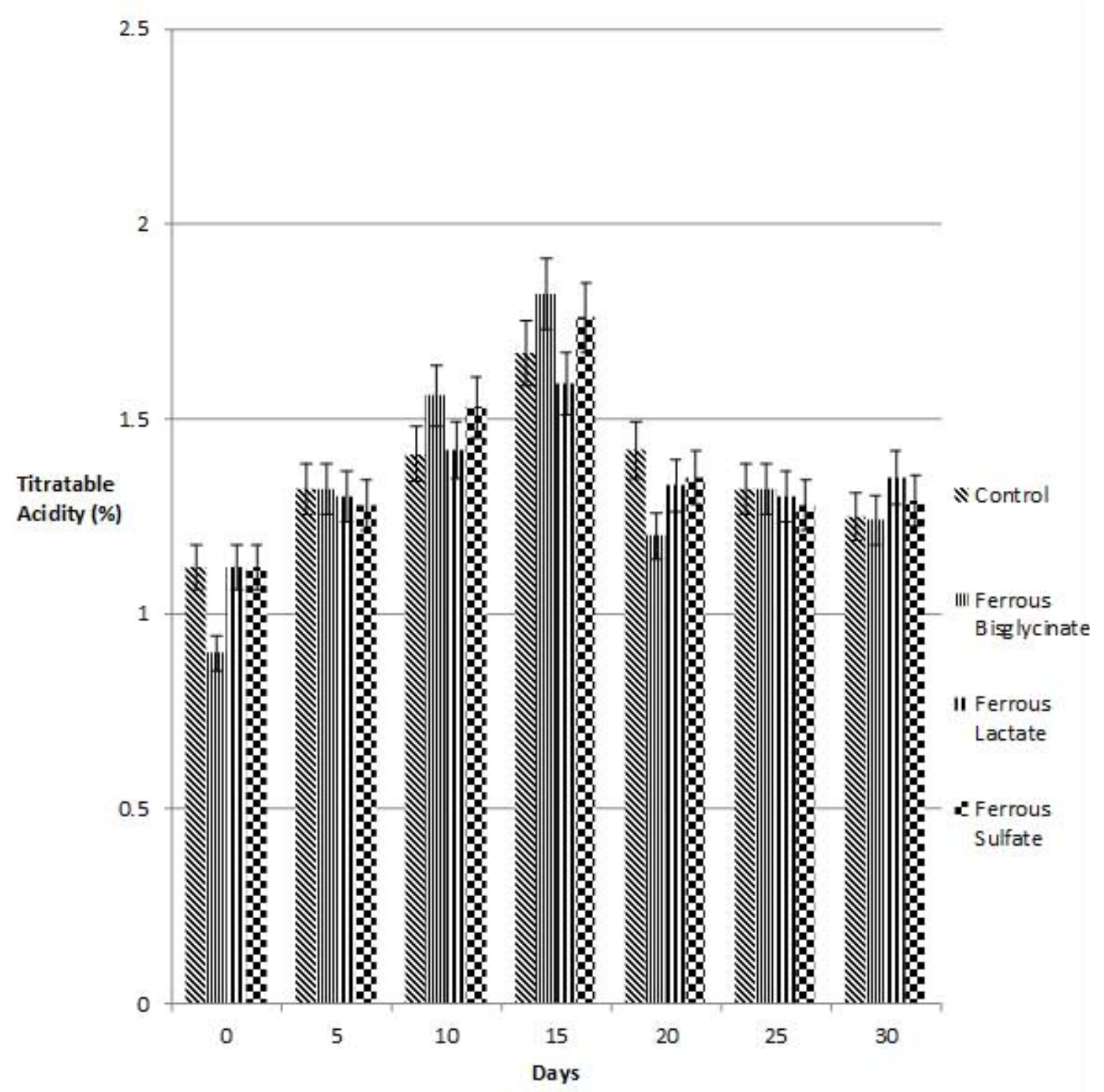

Figure 4: Effects of different iron salts on $\mathrm{pH}$ for yogurt stored at $4^{\circ} \mathrm{C}$ for 30 days.

and the lowest sensory mean scores in all the sensory attributes that were evaluated suggesting a relationship existed between $\mathrm{pH}$ and sensory acceptability of yogurt.

\section{Sensory Evaluation of Pasteurized Milk}

Sensory data in Table 2 shows no significant differences in appearance and flavor among samples at $p<0.05$. This differs with the finding from sensory evaluation of iron fortified yogurt in Table 1 where all attributes evaluated were not significantly different in control yogurt and yogurt fortified with ferrous sulfate microencapsulated. There were no significant differences in flavor in pasteurized liquid milk across all treatments. Milk fortified with ferrous sulfate microencapsulated had significantly lower sensory mean score on taste than any other treatment ( $p$ $<0.05$ ). There were significant differences in overall preference between milk fortified with ferrous sulfate microencapsulated and the rests of the treatments. Overall milk fortified with ferrous sulfate microencapsulate was least preferred especially due to its taste. The panelists were unable to detect significant differences in appearance and flavor between treatments. This is an interesting observation since ferrous bisglycinate is dark in color and it was expected to impact color changes in milk. All milk was rated from like slightly to like moderately in all sensory attributes under study on the hedonic scale.

\section{Chemical Analysis of Lipid Oxidation \\ PV Test for yogurt during storage}

There is a general trend of increasing the PV as storage time increases as shown in figure 6 . But after 10 days of storage PV increased rapidly in yogurt fortified with ferrous sulfate microencapsulated an indication that there was high lipid oxidation. However there was a sharp decrease after 20 days of storage. This decrease is due to decomposition of hydro- 


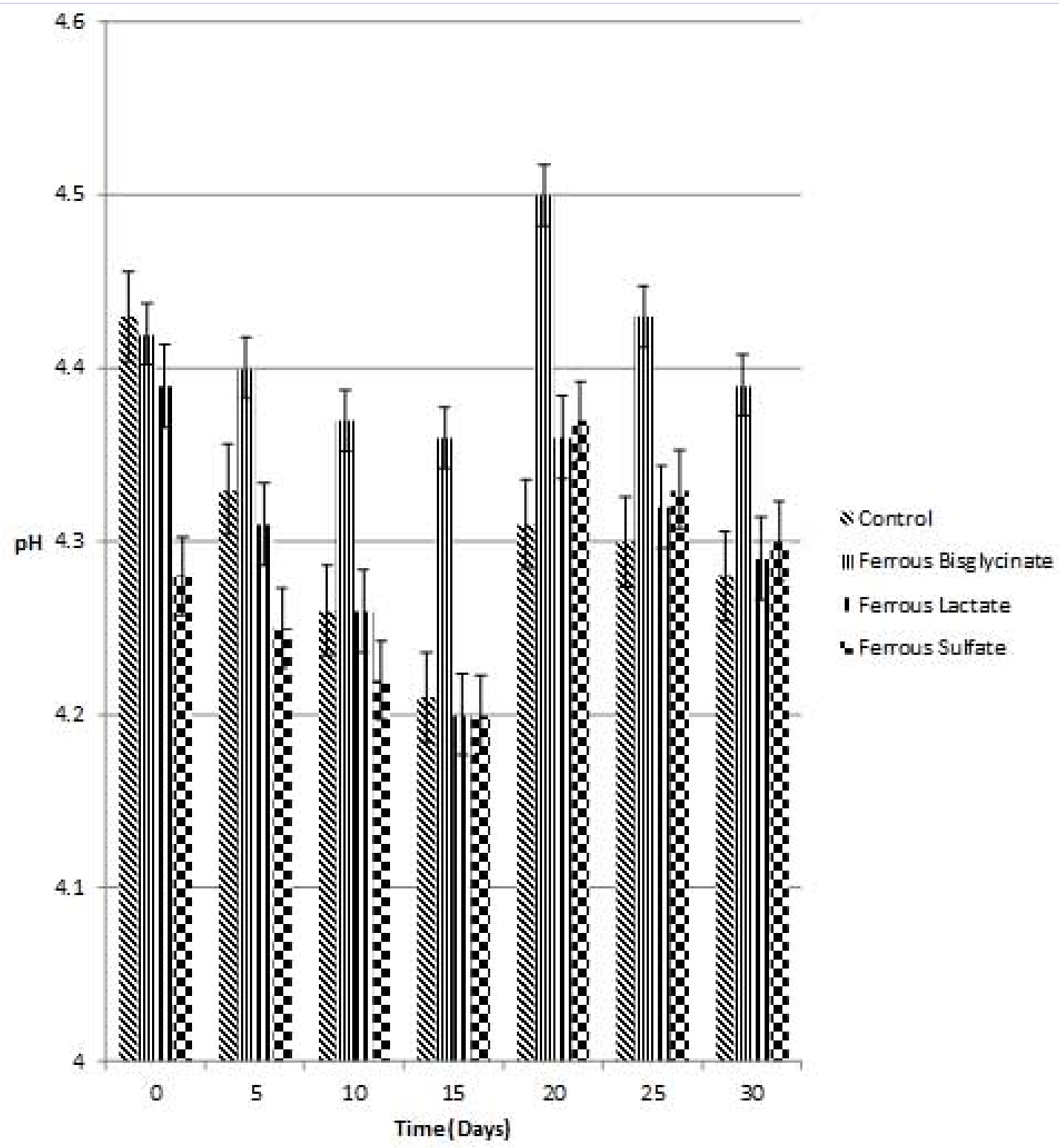

Figure 5: Changes in Titratable Acidity for yogurt fortified with different iron salts and stored at $4^{\circ} \mathrm{C}$ for 30 days.

Table 1: Sensory mean scores of yogurt fortified with different iron salt stored at $4^{\circ} \mathrm{C}$ for 7 days.

\begin{tabular}{|c|c|c|c|c|c|}
\hline Attribute & Control & Ferrous sulfate & Ferrous bisglycinate & Ferrous lactate & $\boldsymbol{P}$-Value \\
\hline Appearance & $6.92^{\mathrm{a}}$ & $6.72^{\mathrm{a}}$ & $5.03^{\mathrm{b}}$ & $5.24^{\mathrm{b}}$ & 0.0001 \\
\hline Body texture & $6.95^{\mathrm{a}}$ & $7.04^{\mathrm{a}}$ & $6.33^{\mathrm{b}}$ & $6.46^{\mathrm{b}}$ & 0.0001 \\
\hline Flavor & $6.67^{\mathrm{a}}$ & $6.66^{\mathrm{a}}$ & $5.63^{\mathrm{b}}$ & $6.00^{\mathrm{b}}$ & 0.0001 \\
\hline Overall acceptance & $6.58^{\mathrm{a}}$ & $6.68^{\mathrm{a}}$ & $5.54^{\mathrm{b}}$ & $5.84^{\mathrm{b}}$ & 0.0001 \\
\hline
\end{tabular}

Means with different superscript within a row are significantly different at $p<0.05, \mathrm{n}=98$

peroxides into secondary products of lipid oxidation. Yogurt fortified with ferrous lactate had the least lipid oxidation. After 23-25 days the control had the highest PV. This may be due to the delay in decomposition of hydroperoxides into secondary products of oxidation as there was no iron to catalyze oxidation process.

\section{PV Test for pasteurized milk in storage}

PV results for pasteurized milk shows that there were significant differences $(p<0.05)$ between treatments after 2 days as shown in figure 7. Control has the lowest PV values throughout the testing period. Milk fortified with ferrous 
Table 2: Sensory mean scores of pasteurized milk fortified with different iron salt stored at $4^{\circ} \mathrm{C}$ for 2 days.

\begin{tabular}{|l|l|l|l|l|}
\hline Attributes & Control & Ferrous bisglycinate & Ferrous Lactate & Ferrous Sulfate \\
\hline Appearance & $7.48 \pm 1.4^{\mathrm{a}}$ & $7.49 \pm 1.42^{\mathrm{a}}$ & $7.20 \pm 1.73^{\mathrm{a}}$ & $7.30 \pm 1.83^{\mathrm{a}}$ \\
\hline Flavor & $6.74 \pm 1.78^{\mathrm{a}}$ & $6.58 \pm 1.65^{\mathrm{a}}$ & $6.67 \pm 1.76^{\mathrm{a}}$ & $6.52 \pm 1.89^{\mathrm{a}}$ \\
\hline Taste & $6.88 \pm 1.74^{\mathrm{a}}$ & $7.08 \pm 1.63^{\mathrm{a}}$ & $6.92 \pm 1.59^{\mathrm{a}}$ & $6.29 \pm 2.25^{\mathrm{b}}$ \\
\hline Texture & $6.52 \pm 1.88^{\mathrm{a}}$ & $7.01 \pm 1.72^{\mathrm{b}}$ & $6.82 \pm 1.78^{\mathrm{b}}$ & $6.59 \pm 2.0^{\mathrm{a}}$ \\
\hline Overall preference & $6.92 \pm 1.48^{\mathrm{a}}$ & $6.99 \pm 1.51^{\mathrm{a}}$ & $6.84 \pm 1.51^{\mathrm{a}}$ & $6.45 \pm 2.12^{\mathrm{b}}$ \\
\hline
\end{tabular}

Means with different superscript in a row are significantly different to each other $(p<0.05), \mathrm{n}=100$

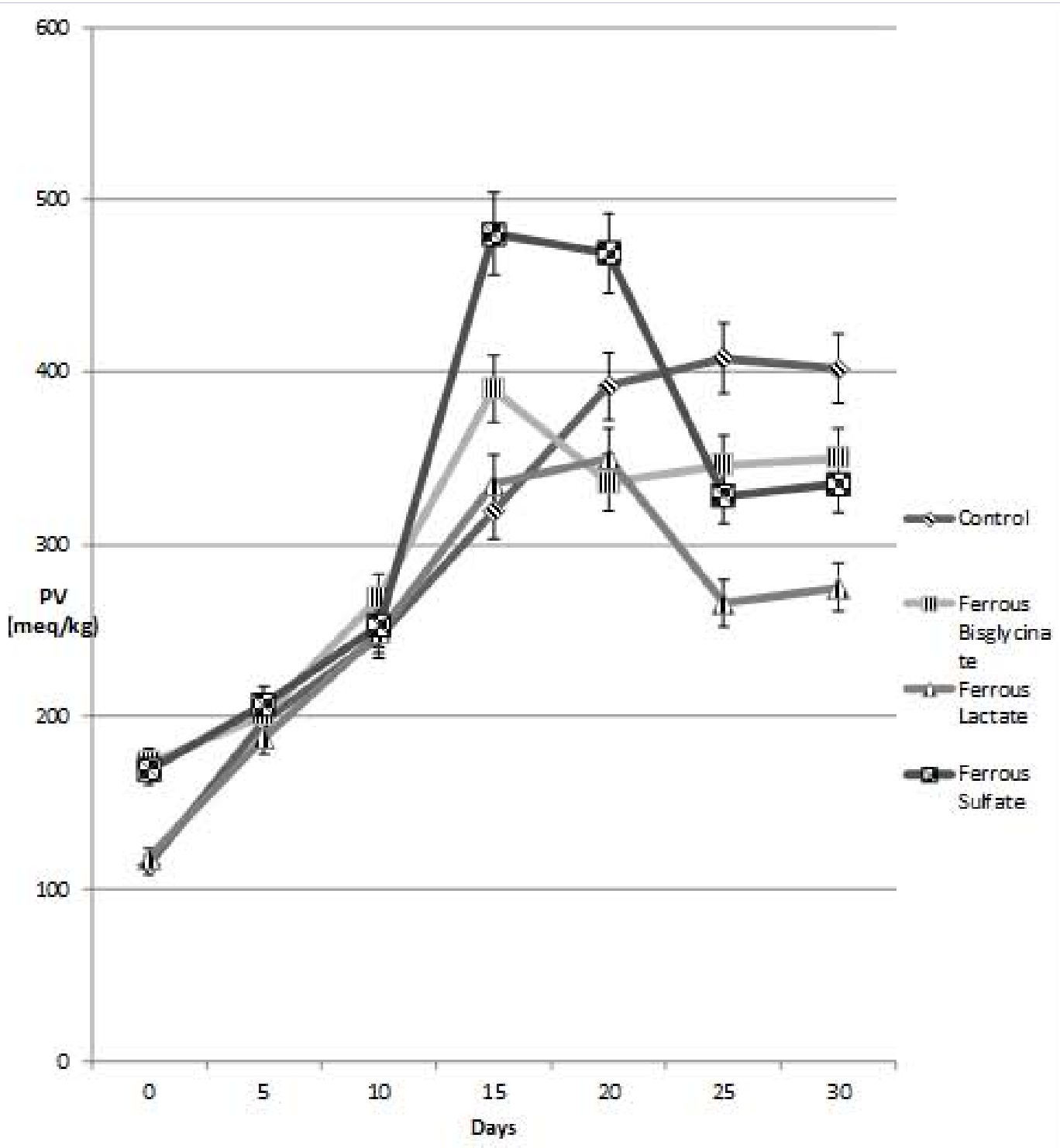

Figure 6: Changes in PV in yogurt fortified with different iron salts and stored at $4^{\circ} \mathrm{C}$ for 30 days.

bisglycinate had high PV values after 12 days. Overall there were no significant differences in mean PV between milk fortified with ferrous bisglycinate and milk fortified with ferrous lactate. After 10 days of storage time yogurt fortified with ferrous sulfate microencapsulated and ferrous bisglycinate had the highest PV values. The pro-oxidant effects of ferrous bisglycinate and ferrous lactate were more pronounced throughout the testing period. The study clearly indicates that iron fortification significantly increases lipid oxidation. Another important observation is that in both cases, PV and TBA tests, ferrous sulfate microencapsulate shows that it enhances lipid oxidation more than other iron salts. The reason behind this behavior is not clear from this 


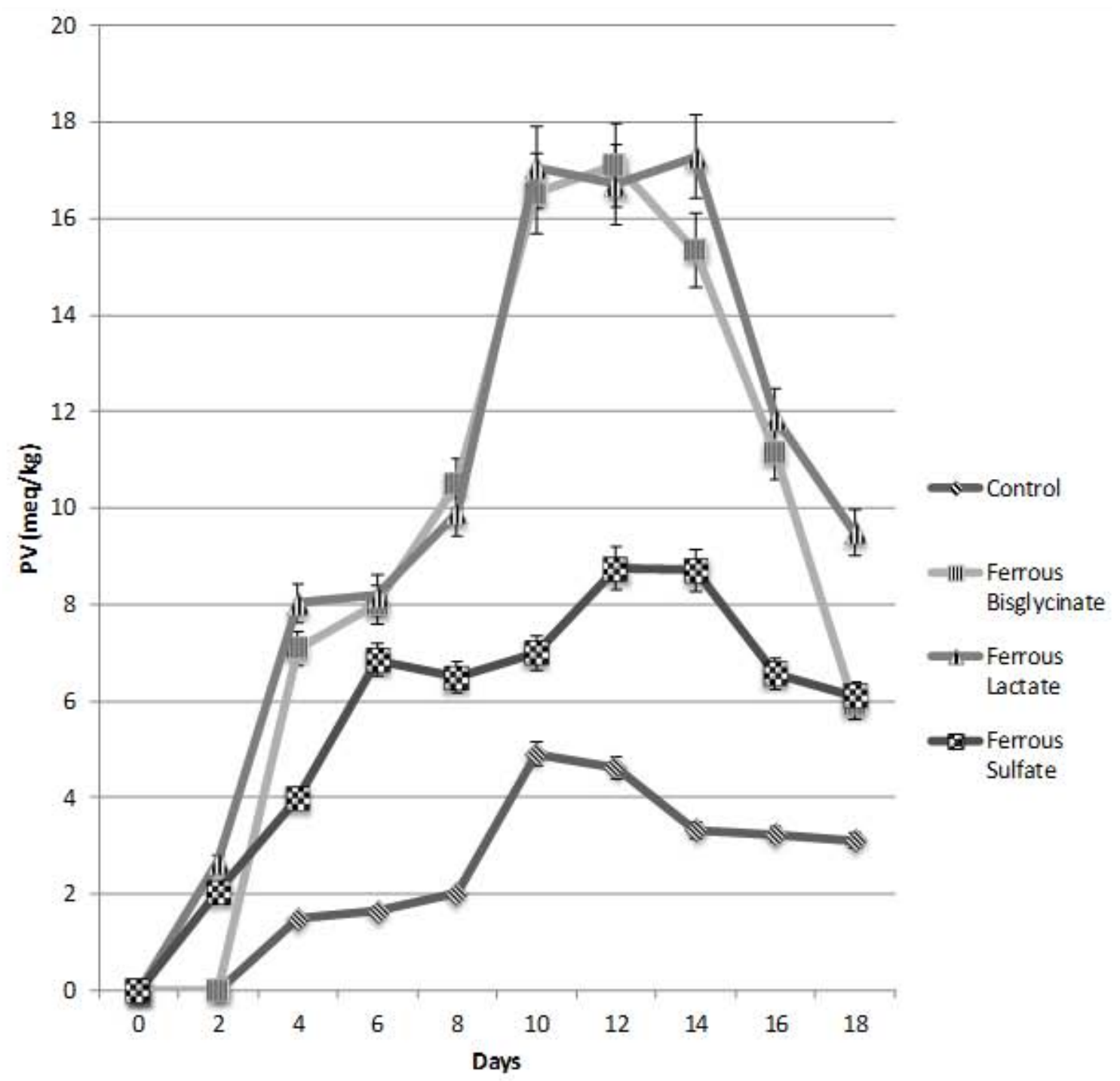

Figure 7: Effects of iron fortification on lipid oxidation in pasteurized milk stored at $4^{\circ} \mathrm{C}$ for 18 days as determined by PV.

study. However it may be concluded that microencapsulation did not provide enough barriers between the iron and milk lipids such that oxidation occurred. This may be due to loss of microencapsulation during homogenization, incubation and microbial growth and activities. This is not conclusive and more studies have to be done on this. This behavior was observed in yogurt and not in pasteurized milk.

\section{TBA Test for Yogurt in Storage}

Data shows that control yogurt had the lowest TBA values as shown by figure 8 and there was generally steady increase in TBA values for all the treatments. Yogurt fortified with ferrous sulfate microencapsulates and yogurt fortified with ferrous bisglycinate had the highest TBA values. TBA reagent when reacted with MDA, a secondary product of lipid oxidation resulting from degradation of hydroperoxide, forms a pink color complex called chromagen which has absorbance at $530 \mathrm{~nm}$. As more of MDA are produced as oxidation continues more chromagen are formed and higher
TBA numbers are obtained at absorbance $530 \mathrm{~nm}$.

Low TBAin control may be due tolow hydroperoxide formation in the early stages of lipid oxidation which consequently resulted into low MDA produced and the subsequent low TBA numbers. Yogurts fortified with ferrous bisglycinate, ferrous lactate and ferrous sulfate microencapsulated had steady increase in TBA which may be due to increased breakdown of hydro peroxides to MDA. Since control had the lowest TBA therefore it can be concluded that all the iron salts under study enhanced lipid oxidation in yogurt.

\section{Conclusion and Recommendations}

All the iron salts studied supported the growth and activities of yogurt starter culture studied though their effects were different for each iron salt. Ferrous bisglycinate supported culture growth the best after 6 hours of incubation. More lactic acid was produced as incubation time increased to 6 hours for 


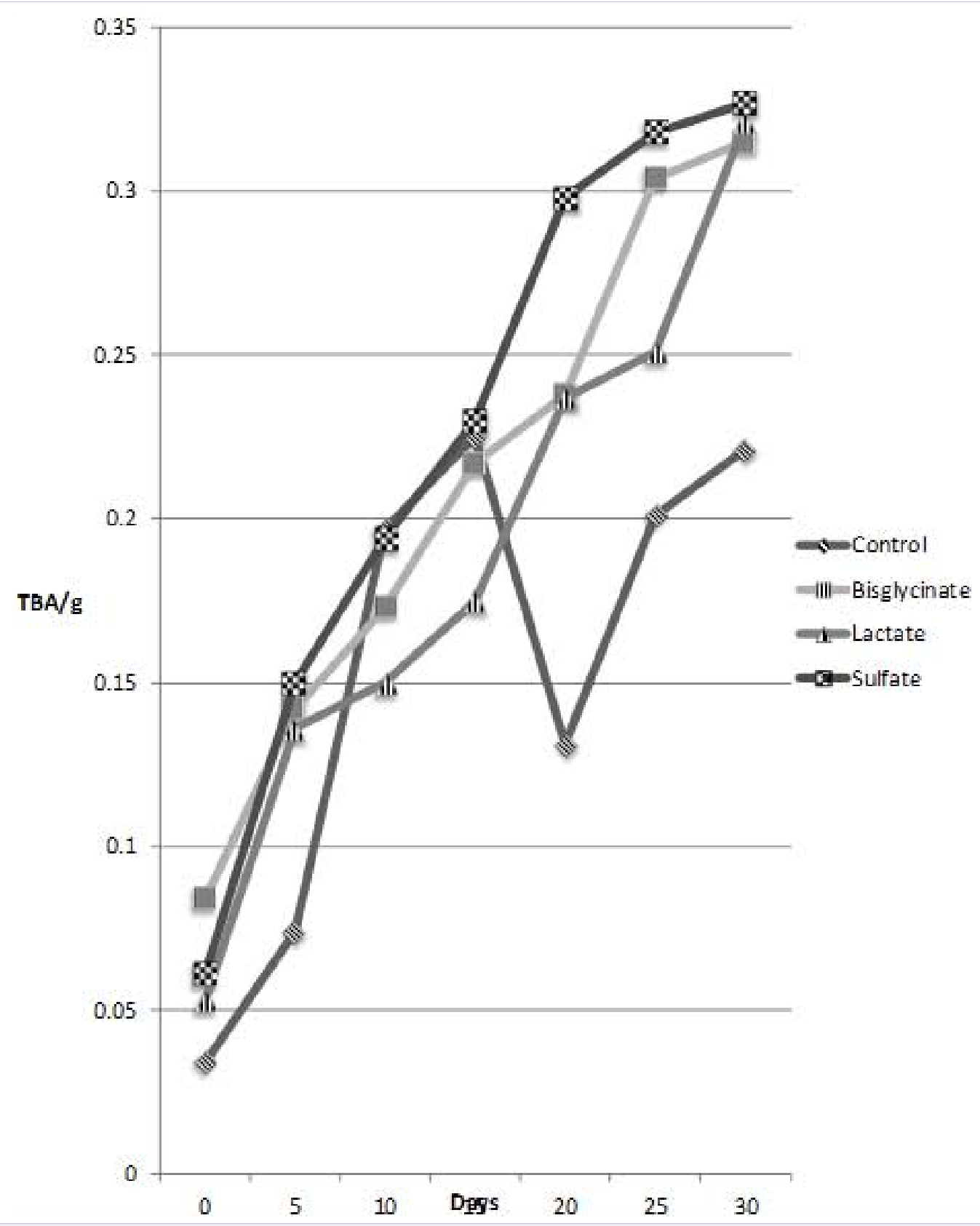

Figure 8: Effect of iron fortification on lipid oxidation in yogurt stored at $4^{\circ} \mathrm{C}$ for 30 days as determined by TBA test.

iron fortified reconstituted skim milk as shown by an increase in TA. In yogurt there was an increase in TA during early days of storage and a decrease in TA towards the end of 30 days storage time.

Consumer acceptance sensory analysis indicated that yogurt fortified with ferrous sulfate microencapsulated were not statistically different from unfortified yogurt in all sensory attributes under study. However, it was statistically higher in sensory acceptability than yogurt fortified with ferrous bisglycinate and ferrous lactate as both gave a darker color which could have been masked by using a more dark food coloring like chocolate.
Pasteurized milk showed statistically insignificant differences in color for all the treatments an indication that the panelist were unable to detect color change as was the case with yogurt. Of all the attributes under study in pasteurized milk, taste was scored the lowest in ferrous sulfate microencapsulated which presumably led to it being preferred the least by panelist.

In both yogurt and pasteurized liquid milk lipid oxidation increased as storage time increased as measured by PV and TBA. Ferrous sulfate fortified yogurt was similar in consumer acceptability to unfortified yogurt in all sensory attributes. However, TBA and PV tests showed higher values in yogurt fortified with ferrous sulfate microencapsulate than in yogurt 
fortified with ferrous bisglycinate and yogurt fortified with ferrous lactate. This may be attributed to increased lipid oxidation as microencapsulation was done using vegetable oil which may also have been oxidized leading to high PV and TBA numbers. Or microencapsulation was destroyed during yogurt mixes homogenization and incubation such that there was free interaction between ferrous sulfate and milk fats leading to high PV and TBA. Therefore, there is a need to establish a reason why ferrous sulfate microencapsulated gave higher PV and TBA (an indication of oxidation) and higher mean sensory score(an indication better sensory quality) because, as would be expected, higher PV and TBA was supposed to go with lower sensory means scores. This study has shown that microencapsulation does not reduce oxidation in yogurt especially when fortification was done before homogenization, pasteurization and incubation but it does reduce oxidation in pasteurized liquid milk.

In this present study bioavailability of fortified iron in yogurt when consumed has not been examined. Therefore another study, in-vitro or in-vivo, would be important to help find out the bioavailability of iron in the body after consuming iron fortified yogurt and milk using animal subjects.

\section{References}

1. Gaucheron F. Iron fortification in dairy industry. Trend in Food Science and Technology. 2001; 11(11): 403-9. doi:10.1016/S09242244(01)00032-2.

2. Martinez-Navarrete N, Camacho MM, Martinez-Lahuerta J, MartinezMonzo J, Fito P. Iron deficiency and iron fortified foods-a review. Food Research International. 2002; 35(2-3): 225-31. doi:10.1016/S09639969(01)00189-2.

3. Van den Broek N, Letsky EA. Etiology of anemia in pregnancy in south Malawi. Am J Clin Nutr. 2000; 72(1 Suppl): 247S-256S

4. World Health Organization and Food and Agriculture Organization of the United Nations. Guidelines on food fortification with micronutrients. Allen L, de Benoist B, Dary 0, Hurrel R, Editors. London: WHO Press. 2009.

5. El-Kholy AM, Osman M, Gouda A, Ghareeb WA. Fortification of Yoghurt with Iron. World Journal of Dairy \& Food Sciences. 2011; 6 (2): 159165.

6. Hurrell R. Preventing iron deficiency through food fortification. Nutr Rev. 1997; 55(6): 210-22.

7. Walter HL, Fanny L, Charles C, Christian R. Minerals and phytic acid interaction: is it a real problem for human nutrition. Int J Food Sc Tech. 2002; 37(7): 727-39.

8. Allen LH, Ahluwalia N. Improving iron status through diet: the applications of knowledge concerning dietary iron bioavailability in human populations. John Snow Incorporated/OMNI Project, Washington: 1997.

9. Hallberg L, Rossander-Hulten L, Brune M, Gleerup A. Inhibition of haem-iron absorption in man by calcium. Br J Nutr. 1993; 69(2): 53340 .

10. Sinnhuber RO, Yu TC. The 2-thiobarbituric acid reaction, an objective measure of the oxidative deterioration occurring in fats and oils. Journal of Japan Oil Chemists Society. 1997; 26: 259-267.

11. Hutkins RW, Nannen NL. pH Homeostasis in Lactic Acid Bacterial. Department of Food Science and Technology University of NebraskaLincoln, Lincoln, 1993.

12. Gibson RS, Perlas L, Hotz C. Improving the bioavailability of nutrients in plant foods at the household level. Proc Nutr Soc. 2006; 65(2): 160-

13. Jay, James M, Loessner, Martin J, Golden, David A. Modern Food Microbiology. 7th Edn. Springer US Publishers, London: 2005.

14.Wambugu SM, Taylor JRN, Dewar J. Effect of addition of malted and fermented sorghum flours on proximate composition, viscosity, $\mathrm{pH}$ and consumer acceptability of extruded sorghum weaning porridges. 2002.

15. Haile M. Iron Fortification Technology Development: New Approaches. J Nutr. 2006; 136(4): 1059-63. 